\title{
CONSEQUENCES OF PORT MISMATCH AND FINITE ISOLATION ON POWER COMBINING.
}

\author{
K. W. Eccleston \\ Department of Electrical Engineering \\ National University of Singapore
}

\begin{abstract}
In solid-state power amplification, power combining is an important issue since the output powers from individual transistors are too small for many transmission applications. An important aspect of power combining is the combining efficiency being the ratio of the power delivered to the load to that available from the sources connected to the input. In a practical power combiner, waves incident on the input ports are scattered to all ports including the output port. This paper will discuss the effects of port mismatch and finite combiner isolation on combining efficiency. For a lossless power combiner, it is shown that the efficiency may be optimised by optimising the matching at the combiner output.
\end{abstract}

\section{INTRODUCTION.}

Due to the power limitations of microwave transistors, power combining is necessary when high output powers are required. In the conventional approach, the output powers of several amplifier modules are combined in parallel. This approach has the added advantage of graceful degradation when amplifier modules fail. The current trend is the realisation of planar combining structures in microstrip which allow the integration of the amplifier modules, output combiner and input divider. It is of interest to consider the combining efficiency, which is the ratio of the load power to the total power available from the amplifier outputs, as a figure of merit. It is the purpose of this paper to examine the effect of port mismatch and finite combiner input- port isolation, on the combining efficiency.

In recent years there have appeared a number of realisations of solid-state power amplifiers (SSPAs) which use parallel combining techniques to obtain output powers of the order tens of Watt [1] - [3]. The common feature of these realisations is that they use loss-less power dividers and combiners. ie. the no isolation resistors are used. As a consequence of the unitary properties of a loss-less networks, input reflection coefficients and inter-input-port couplings are considerable. Indeed when a large number of amplifiers are combined, the magnitude of the inter-input-port couplings are comparable with the input to output transmission. Further, studies have indicated that the amount of input-port isolation that can be obtained when isolation resistors are used is limited. eg. references [4] and [5]. Therefore the study of the impact of finite input-port isolation and mismatch is justified.

The following is a brief review of the previous work that has been reported by other researchers on the issue of power combining. Ernst et al [6] have reported the graceful degradation properties of matched-isolated parallel combined power amplifiers. Following from this study, Saleh [7] proposed two methods for improving the graceful degradation performance of parallel combined power amplifiers. Galani et al [8] developed an approximation for the forward transmission of a parallel combined amplifier in terms of amplifier, divider and combiner s-parameters. In that paper they analyzed amplifier performance in the presence of small variations in amplifier parameters and amplifier failure and presented some design guidelines. In a recent paper, Gupta [9] has derived expressions for the worst case combining efficiency as a function of signal imbalance. In another paper, Gupta [10] has shown how the combining efficiency may be optimised by adjustment of the input signal amplitudes and phases. In these approaches Gupta assumed that amplifier, load and combiner port reflection coefficients were zero [9][10]. Under this assumption, the output power is only a function of input signal values, and combiner forward transmission S-parameters.

\section{COMBINER SCATTERING ANALYSIS}

In this section a scattering analysis is performed on a power combining system depicted in figure 1. This system consists of a n-way power combiner, a load and $\mathrm{n}$ sources connected at the input which have finite impedance mismatch.

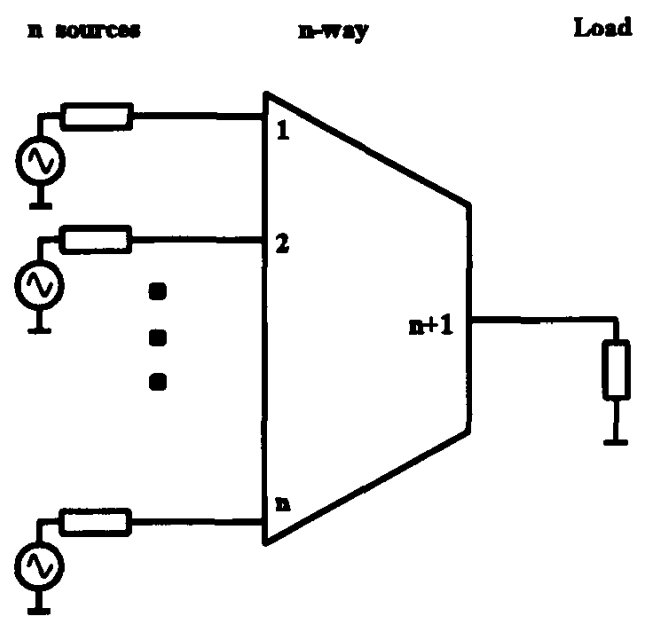

Figure 1 Combiner system 
The n-way power combiner is represented by its S-matrix, $\mathbf{S}$, of order $\mathrm{n}+1$ with the input ports numbered 1 to $\mathrm{n}$ and the output port being numbered $n+1$. The waves incident and scattered at the ports of the combiner are related via the $S$ matrix:

$$
\mathbf{b}=\mathbf{S} \mathbf{a}
$$

where $\mathbf{a}$ and $\mathbf{b}$ are the vectors, of dimension $n+1$, representing the incident and scattered waves at each port of the combiner respectively. The reflection coefficient of the termination at the $i^{\text {th }}$ port is $\Gamma_{\mathrm{i}}$. The excitation wave at the $\mathrm{i}^{\text {th }}$ input port is $\mathrm{e}_{\mathrm{i}}$, and it assumed that there is no excitation at the output. Thus the power delivered into a non-reflecting load by the $i^{\text {th }}$ source is $\left|e_{i}\right|^{2}$. Therefore the incident wave on the $\mathrm{i}^{\mathrm{it}}$ port of the combiner is given by:

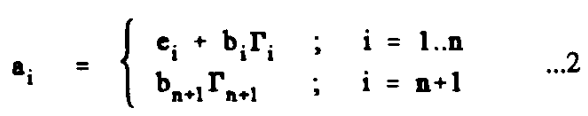

Let $\mathbf{e}$ be a vector of dimension $n+1$ representing the excitations, with $e_{n+1}$ equal to zero. Let $\Gamma$ be a square matrix of order $n+1$ in which all entries are zero except for those on the leading diagonal whose values are the termination reflection coefficients. ie the $\mathrm{i}^{\text {th }}$ entry on the leading diagonal is $\Gamma_{\mathrm{i}}$. Therefore:

$\mathbf{n}=\boldsymbol{\Gamma} \mathbf{b}+\dot{0}$

Substitution of equation 3 into 1 and re-arranging yields:

$$
\mathbf{b}=(\boldsymbol{U}-\mathbf{S} \Gamma)^{-1} \mathbf{S}
$$

where $\mathbf{U}$ is the identity matrix of order $n+1$. The power delivered to the load is given by:

$$
P_{\text {Lond }}=\left|b_{n+1}\right|^{2}\left(1-\left|\Gamma_{n+1}\right|^{2}\right) \quad \ldots .5
$$

and the total power available from the amplifiers is given by:

$$
P_{\mathbb{D N} \text {-Amilebie }}=\sum_{i=1}^{n} \frac{\left|e_{i}\right|^{2}}{1-\left|\Gamma_{i}\right|^{2}} \quad \ldots 6
$$

Hence the combining efficiency is:

$$
\eta_{C}=\frac{P_{\text {Lood }}}{P_{\mathrm{DN} \text {-Amileble }}}
$$

It is clear from this general analysis, that $\eta_{c}$ is dependent on all the S-parameters of the combiner, as well as the load reflection coefficient and amplifier output characteristics. If $\Gamma$ is identically zero, then the combining efficiency is only dependent on the input-port to output-port transmissions.

For practical use, equation 7, and equations 4 - 6 that are associated with it, will require simplification. In the simplification that follows, it will be assumed that: i) the transmission of waves between the input and output ports are all equal to a complex parameter $T$, and ii) the load connected at the input has zero reflection coefficient. In practice both these assumptions are reasonable.

The scattering parameter matrix of the power combiner may be partitioned into four blocks:

$$
s=\left[\begin{array}{ll}
s^{11} & s^{12} \\
s^{21} & s^{22}
\end{array}\right]
$$

where $S^{\prime \prime}$ is square matrix of order $n$ and represents the combiner input mismatches and finite coupling between the input ports, $\mathbf{S}^{12}$ is a column vector of dimension $n$ and represents the transmission of waves from the output port to the input ports, $\mathrm{S}^{21}$ is row vector of dimension $\mathrm{n}$ and represents the transmission of waves from the input ports to the output ports, and finally $\mathbf{S}^{22}$ is the output reflection coefficient and is equal to $S_{n+1, n+1}$. When the combiner is reciprocal, $\mathbf{S}^{11}$ is symmetric and $\mathbf{S}^{21}$ is the transpose of $\mathbf{S}^{12}$.

Based on the assumptions stated earlier:

$$
\mathbf{s}^{21}=\left[\mathbf{s}^{12}\right]^{\mathrm{T}}=\mathrm{T}\left[\begin{array}{lllllll}
1 & 1 & 1 & 1 & \ldots & 1
\end{array}\right] \ldots 9
$$

and:

$$
U-s \Gamma=\left[\begin{array}{cc}
U^{11}-s^{11} \Gamma^{11} & Z \\
-s^{21} \Gamma^{11} & 0
\end{array}\right] \ldots 10
$$

where $\mathbf{Z}$ is a zero vector of dimension $n, \Gamma^{11}$ is a square matrix of order $n$ which is zero with the exception of the entries on the leading diagonal which are the reflection coefficients of the sources connected at the input, and $\mathbf{U}^{\prime \prime}$ is the identity matrix of order $\mathrm{n}$. Further assume that all input excitation waves are equal, and for convenience, equal to unity. ie:

$$
=\left[\begin{array}{lllllll}
1 & 1 & 1 & 1 & \ldots & 1 & 0
\end{array}\right]^{\mathrm{T}}
$$

Therefore:

$$
\text { se }=\left[\begin{array}{lllll}
\beta_{1} & \beta_{2} & \cdots & \beta_{n} & n T
\end{array}\right]^{T}
$$

where:

$$
\beta_{i}=\sum_{j=1}^{n} s_{i j}
$$

The significance of $\beta_{i}$ will become more apparent later when the properties of a loss-less power combiner are considered. Equations 9 to 12 may be substituted into equation 4 . After re- 
arranging and decoupling the bottom row of the resulting matrix equation, the result is:

$$
b_{n+1}=T\left(n+\sum_{j=1}^{n} b_{j} \Gamma_{j}\right)
$$

and:

$$
\left(\widetilde{J}^{11}-s^{11} \Gamma^{11}\right)\left[\begin{array}{c}
b_{1} \\
b_{2} \\
\cdot \\
\cdot \\
b_{n}
\end{array}\right]=\left[\begin{array}{c}
\beta_{1} \\
\beta_{2} \\
\cdot \\
\cdot \\
\beta_{n}
\end{array}\right]
$$

Therefore $b_{n+1}$ can be found by determining $b_{1} \ldots b_{n}$ from the solution of equation 15 . Note that when all sources are matched, the load power will be (nT) ${ }^{2}$ and hence efficiency will be $\mathrm{T}^{2}$. Equation 14 indicates that the wave emanating from the output port will consist of a desired component $\mathrm{nT}$ and a component that is a perturbation due to source mismatch.

To estimate the lower bound for efficiency, the upper bound for the input available power and the lower bound for the output power are required. From equation 14 it is clear that:

$$
\left|b_{n+1}\right| \geq|T|\left(n-P_{-m} \sum_{j=1}^{i}\left|b_{j}\right|_{-\infty}\right)
$$

where $\rho_{\max }$ is the maximum magnitude of the source reflection coefficients. It is easy to show from equation 6 and assuming that the input excitation waves are all equal to unity:

$$
P_{\mathrm{DN}-\text { Anilable }} \leq \frac{n}{1-P_{\max }^{2}}
$$

The next step is to estimate the upper bound for the magnitudes of the waves reflected from the input ports of the combiner.

Equation 15 can be rewritten:

$$
b^{\prime}=\left(\mathbf{U}^{11}-\mathbf{G}\right)^{-1} \boldsymbol{\beta}
$$

where $\mathbf{b}^{\prime}$ is the vector of dimension $n$ containing $b_{1} \ldots b_{n}, \beta$ is the vector of dimension of $n$ containing $\beta_{1} \ldots \beta_{n}$, and $G$ is equal to $\mathbf{S}^{\prime \prime} \Gamma^{\prime \prime}$. Provided that the norm of $\mathbf{G}$ is sufficiently small, then a binomial expansion of the right-hand side of equation 18 may be used [8]:

$$
b^{\prime}=\left(\mathbf{U}^{11}+\mathbf{G}+\mathbf{G}^{2}+\mathbf{G}^{3} \ldots\right) \boldsymbol{\beta} \ldots 19
$$

It is therefore apparent that $\mathbf{b}^{\prime}$ is equal to $\beta$ plus some perturbation due to the presence of non zero $S^{11}$ and $\Gamma^{\prime \prime}$.
It may be recalled from matrix algebra that if $\mathbf{A}$ and $\mathbf{B}$ are matrices; then the $\mathrm{ij}^{\text {th }}$ element of $\mathbf{A B}$ is in fact the dot product of the $i^{\text {th }}$ row of $A$ with the $j^{\text {th }}$ column of $B$. Likewise, if $v$ is a column vector; then the $i^{\text {th }}$ element of product $A \mathbf{v}$ is the dot product of the $i^{\text {th }}$ row of $A$ and $v$. Thus equation 19 can be written as:

$$
\mathbf{b}_{j}=\boldsymbol{\beta}_{j}+\dot{\boldsymbol{\Sigma}} \operatorname{row}\left(\mathbf{G}^{\mathbf{m}} \mid j\right) \cdot \boldsymbol{\beta}
$$

Let $\sigma_{i}$ be the Euclidean vector norm of the $i^{\text {th }}$ row or column of $S^{\prime \prime}$, which is given by:

$$
\sigma_{i}=\sqrt{\sum_{j=1}^{n}\left|s_{i j}\right|^{2}}=\sqrt{\sum_{j=1}^{n}\left|s_{j i}\right|^{2}}
$$

Based on Euclidean vector norms and then with the application of the Schwarz inequality, it can be shown that (see Appendix A):

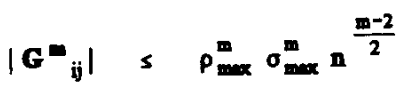

for $m$ is greater than unity and $o_{\max }$ is the maximum value of the Euclidean row vector norms of $\mathbf{S}^{11}$. Thus the magnitude of the Euclidean row norm is bounded:

$$
\left|\operatorname{row}\left(\mathbf{G}^{\mathbf{m}} \mid \mathbf{i}\right)\right| \leq \begin{cases}\rho_{\max } \sigma_{\max } & ; m=1 \\ \left|G^{-}\right|_{i j \max } \sqrt{n} ; m>1\end{cases}
$$

Let $\beta_{\max }$ be the magnitude of the largest element of $\beta$, then the upper bound for the Euclidean norm of $\beta$ will be:

$$
|\boldsymbol{\beta}| \leq \boldsymbol{\beta}_{\max } \sqrt{n}
$$

With the application of the Schwarz inequality and the use of equations 22 to 24 :

$$
\left|\operatorname{row}\left(G^{m} \mid j\right) \cdot \beta\right| \leq \beta_{\max } \rho_{\max }^{m} \sigma_{\max }^{m} n^{\frac{m}{2}} \ldots 25
$$

Based on this result and equation 20, the upper bound on the magnitude of $b_{i}$ is given by the series:

$$
\left|b_{j}\right| \leq \beta_{\max } \sum_{m_{\infty}}^{\infty} \rho_{\max }^{m} \sigma_{\max }^{m} n^{\frac{m}{2}}
$$

for $\mathrm{j}=1$..n. Which may be written in closed form as:

$$
\left|b_{j}\right| \leq \frac{\beta_{\max }}{1-p_{\max } \sigma_{\max } \sqrt{n}}
$$


provided $\rho_{\max } \sigma_{\max } n^{1 / 2}$ is less than unity. Finally after combining the results of equations 16,17 and 27 , the lower bound for combining efficiency is obtained:

$$
\eta_{0} 2 n|T|^{2}\left(1-\rho_{\max }^{2}\right)\left(1-\frac{\beta_{\max } \rho_{\max }}{1-\rho_{\max } \sigma_{\max } \sqrt{n}}\right)^{2}
$$

\section{DISCUSSION.}

Equation 28 indicates the lower bound for combining efficiency in the presence of source impedance mismatch, and non-ideal combiner behaviour. It is thus apparent that efficiency is maximised when $\rho_{\max }, \sigma_{\max }$ and $\beta_{\max }$ are minimised, and $n|T|^{2}$ approaches unity. Since $\beta_{i}$ is equal to the dot product of the $i^{\text {th }}$ row or column of $S^{\prime \prime}$ and the vector $\left[\begin{array}{llllll}1 & 1 & 1 & \ldots & 1\end{array}\right]$, then according to the Schwarz inequality it can be said that:

$$
\beta_{\max } \leq \sigma_{\max } \sqrt{n}
$$

But a more interesting result occurs when the combiner is assumed lossless which is reasonably valid for SSPA realisations that have been reported in the literature [1] - [3]. Under this condition the unitary properties of the $S$ matrix hold [11]. If it is assumed that the transmissions between the input ports and the output port are equal, then with the application of the unitary properties [11], the following can be said for this lossless combiner:

$$
\begin{gathered}
\beta_{\max }=\rho_{0} \\
|T|=\sqrt{\frac{1-\rho_{0}^{2}}{n}}
\end{gathered}
$$

and

$$
\sigma_{\max }=\sqrt{\frac{n-1+p_{0}^{2}}{n}}
$$

where $\rho_{0}$ is the magnitude of the output reflection coefficient of the combiner. After substition of equations 30 to 32 into equation 28 the result for the efficiencey for a lossless combiner is:

$$
\begin{aligned}
\eta_{0-L a n d e x}= & \left(1-p_{0}^{2}\right)\left(1-p_{\max }^{2}\right) \cdot \\
& \cdot\left(1-\frac{p_{0} p_{\max }}{1-p_{\max } \sqrt{1-1+p_{0}^{2}}}\right)^{2}
\end{aligned}
$$

Thus for the lossless power combiner equation 33 indicates that efficiency can be maximised by minimising the output reflection coefficient of the combiner. In fact the output reflection coefficient of a lossless power combiner can be made negligible over a narrow bandwidth. Equation 33 is useful in SSPA system design as it can be used to determine either the maximum amplifier module output impedance mismatch that can be tolerated for a given $n$-way power combiner, or the alternatively the maximum number of amplifier modules that can be combined for a given amplifier specification and combiner output mismatch.

Further for the lossless case, equation 27 indicates that the waves reflected from the input ports is minimised when the combiner output mismatch is minimised and in the extreme, the sources see matched loads.

\section{CONCLUSION.}

This paper has shown the development of an expression for the lower bound of the combining efficiency which takes into account source impedance mismatch, combiner port impedance mismatch and finite input-port isolation. It was assumed that: that the sources have equal excitation, the combiner is reciprocal, the load connected to the combiner output has zero reflection, and that the combiner input to output transmissions are identical. The latter three assumptions are reasonable in practice and the former assumption can in practice be established by circuit trimming. It was shown that in the case of the lossless combiner, combining efficiency can be maximised by minimising its output reflection coefficient. This later result is of practical importance since recently reported SSPAs have utilised lossless power combiners. The expression developed for the efficiency of combining using a lossless power combiner is useful in SSPA system design calculations.

The work that remains is to apply the above analysis techniques to the complete parallel combined amplifier system which includes the input power divider.

\section{APPENDIX A}

\section{Derivation of Equation 22.}

Equation 22 gives the upper bound for the magnitude of the $\mathrm{ij}^{\text {th }}$ element of $\mathbf{G}^{\mathrm{m}}$ where $\mathrm{m}$ is some integer greater than one. Since $\mathbf{G}^{\mathbf{m}}$ is equal to the product $\mathbf{G}^{\mathbf{m} \cdot \mathbf{1}} \mathbf{G}$, then by definition of matrix products:

$$
\mathbf{G}_{\mathrm{ij}} \quad=\operatorname{row}\left(\mathbf{G}^{m-1} \mid \mathrm{i}\right) \cdot \operatorname{col}(\mathbf{G} \mid \mathrm{j}) \quad \ldots \mathrm{Al}
$$

which is the dot product of the $\mathrm{i}^{\text {th }}$ row or $\mathbf{G}^{\mathrm{m}-1}$ with the $\mathrm{j}^{\text {th }}$ column of $\mathbf{G}$. From the Schwarz inequality:

$$
\left|\mathbf{G}^{\mathbf{m}} \mathbf{j}_{\mathbf{j}} \leq\right| \operatorname{row}\left(\mathbf{G}^{\mathbf{m}-1} \mid \mathrm{i}\right)|| \operatorname{col}(\mathbf{G} \mid \mathrm{j}) \mid \quad \ldots \mathrm{A} 2
$$


It can be easily shown that the upper bound for the Euclidean norms of either of the rows or columns of $\mathbf{G}$ is:

$$
|\operatorname{row}(\mathbf{G} \mid \mathbf{i})|_{\max }=|\operatorname{col}(\mathbf{G} \mid j)|_{\max }=\rho_{\max } \sigma_{\max }
$$

From equation 23:

$$
\left|\operatorname{row}\left(\mathbf{G}^{m-1} \mid \mathbf{i}\right)\right| \leq\left|\mathbf{G}^{\mathbf{m}-1}\right|_{\max } \sqrt{\mathbf{n}}
$$

Substitution of equation $\mathrm{A} 3$ and $\mathrm{A} 4$ into $\mathrm{A} 2$ results in:

$$
\left|G^{*}{ }_{i j}\right| \leq\left|G^{-1}\right|_{\text {max }} P_{\max } \sigma_{\max } \sqrt{n} \quad \ldots A 5
$$

Likewise it follows from equation A5 that:

$$
\left|\mathbf{G}^{\boldsymbol{D}-1}\right|_{i j} \leq\left|\mathbf{G}^{\mathbf{m}-2}\right|_{\operatorname{mx}} \boldsymbol{\rho}_{\max } \sigma_{\max } \sqrt{\mathbf{n}} \quad \ldots \text { A6 }
$$

Substituting equation A6 into equation A5 yields:

$$
\left|G_{i j}^{m}\right| \leq\left|G^{m-2}\right|_{\max } P_{\max }^{2} o_{\max }^{2}(\sqrt{n})^{2}
$$

It thus follows that

$$
\left|G^{=}\right|_{i j} \leq\left|G^{=-k}\right|_{\max } P_{\max }^{k} \sigma_{\max }^{k}(\sqrt{n})^{k}
$$

for $\mathrm{m}-\mathrm{k}$ greater than unity. From equations $\mathrm{A} 2$ and $\mathrm{A} 3$ :

$$
\left|G_{i j}^{2}\right| \leq P_{\max }^{2} \sigma_{\max }^{2}
$$

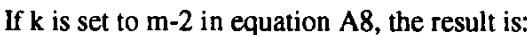

$$
\left|G_{i j}^{m}\right| \leq\left|G_{i j}^{2}\right|_{\max } P_{\max }^{m-2} \sigma_{\max }^{m-2}(\sqrt{n})^{m-2}
$$

Substition of equation $\mathrm{A} 9$, into equation $\mathrm{A} 10$ results in equation 22.

\section{REFERENCES:}

[1] H. Mizuno, H. Mitsumoto \& N. Yazawa, "A $12.5 \mathrm{GHz}-$ Band 50W Solid-State Power Amplifier for Future Broadcasting Satellites", 1990 International Microwave Symposium, 1990 IEEE MTT-S, pp1337 - 1340.

(2) P. Martin, P. J. Allen \& J. B. Ness, "A $14 \mathrm{GHz} 80$ Watt GaAs FET Amplifier", $21^{\text {t }}$ European Microwave Conference, Sept. 1991, pp1465 - 1468.

[3] R. Mallavarpu \& M. P. Puri, "High Power $14 \mathrm{GHz}$ SSPA for Satcom Applications", 1993 International Microwave Symposium, 1993 IEEE MTT-S, pp1217 - 1220.
14] Z. Galani \& S. J. Temple, "A Broadband Planar Nway Combiner/Divider", 1977 International Microwave Symposium, 1977 IEEE MTT-S, pp499 - 502.

[5] M. Miyazaki, O. Ishida \& T. Hashimoto, "N-Way Sectorial Hybrid Power Divider Design Using Boundary Element Method", International Journal of Microwave and Millimeter-Wave Computer-Aided Engineering, Vol. 3, No. 3, 1993, pp175-182.

[6] R. L. Ernst, R. L. Camisa \& A. Presser, "Graceful Degradation Properties of Matched N-port Power Amplifier Combiners", 1977 International Microwave Symposium, MTT-S 1977, pp174 - 177.

[7] A. A. M. Saleh, "Improving the Graceful-Degradation Performance of Combined Power Amplifiers", IEEE Transactions on Microwave Theory and Techniques, Vol. 28, No. 10, October 1980, ppl068 -1070.

[8] Z. Galani, J. L. Lampen \& S. J. Temple, "SingleFrequency Analysis of Radial and Planar Amplifier Combiner Circuits", IEEE Transactions on Microwave Theory and Techniques, Vol. 29, No. 7, July 1981, pp642 -654 .

[9] M. S. Gupta, "Degradation of Power Combining Efficiency Due to Variability Among Signal Sources", IEEE Trans. on Microwave Theory and Techniques, Vol. 40, No 5, May 1992, pp1031 - 1034.

[10] M. S. Gupta, "Power combining efficiency and its optimisation", IEE Proc. Pt H, Vol. 139, No.3, June 1992, pp233 - 238.

[11] R. E. Collin, Foundations for Microwave Engineering, 2nd Ed., McGraw-Hill 1992. pp251 - 253. 